\title{
SIDIS transverse spin azimuthal asymmetries at COMPASS: Multidimensional analysis
}

\author{
Bakur Parsamyan ${ }^{* \dagger}$ \\ University of Turin and Torino Section of INFN \\ Via P. Giuria 1, 10125 Torino, Italy \\ E-mail: bakur.parsamyan@ern.ch
}

\begin{abstract}
Exploration of transverse spin structure of the nucleon via study of the spin (in)dependent azimuthal asymmetries in semi-inclusive deep inelastic scattering (SIDIS) and Drell-Yan (DY) reactions is one of the main aspects of the broad physics program of the COMPASS experiment (CERN, Switzerland). In past decade COMPASS has collected a considerable amount of polarized deuteron and proton SIDIS data, while recent 2014 and 2015 runs were dedicated to the Drell-Yan measurements. Results on SIDIS azimuthal effects provided so far by COMPASS play an important role in general understanding of the three-dimensional nature of the nucleon. Giving access to the entire "twist-2" set of transverse momentum dependent (TMD) parton distribution functions (PDFs) and fragmentation functions (FFs) COMPASS data are being widely used in phenomenological analyses and experimental data fits. Recent unique and first ever $\mathrm{x}-Q^{2}$-z-pT multidimensional results for transverse spin asymmetries obtained by COMPASS serve as a direct and unprecedented input for one of the hottest topics in the field of spin-physics: the TMD $Q^{2}$ evolution related studies. In addition, extraction of the Sivers and all other azimuthal effects from first ever polarized Drell-Yan data collected recently by COMPASS will reveal another side of the spin-puzzle clarifying the link between SIDIS and Drell-Yan branches. This will be a unique possibility to test predicted universality and key-features of TMD PDFs using essentially the same experimental setup and exploring the same kinematical domain. In this review main focus will be given to the very recent results from COMPASS multi-dimensional analysis of transverse spin asymmetries and to the physics aspects of COMPASS polarized Drell-Yan program.
\end{abstract}

QCD Evolution 2015 -QCDEV2015-

26-30 May 2015

Jefferson Lab (JLAB), Newport News Virginia, USA

* Speaker.

${ }^{\dagger}$ on behalf of the COMPASS collaboration. 


\section{Introduction}

Schematic view of the SIDIS framework and some notations and definitions adopted in this letter such as coordinate system, azimuthal angles, etc. are presented in Figure 1. In this framework the target transverse polarization $\left(S_{T}\right)$ is defined relative to the virtual photon momentum direction, which is the most natural basis from the theoretical point of view. However, in experiment target is being polarized in laboratory system and transverse polarization $\left(P_{T}\right)$ is defined relative to the beam (incoming lepton) direction. As it was demonstrated in [1]-[4] this difference influences azimuthal distributions in the final state. After applying appropriate conversions, the model-independent expression for the SIDIS cross-section for transversely (w.r.t. lepton beam) polarized target can be re-written in the following way [1]-[4]:

$$
\begin{aligned}
& \frac{d \sigma}{d x d y d z p_{T}^{h} d p_{T}^{h} d \phi_{h} d \phi_{S}}=2\left[\frac{\cos \theta}{1-\sin ^{2} \theta \sin ^{2} \phi_{S}}\right]\left[\frac{\alpha}{x y Q^{2}} \frac{y^{2}}{2(1-\varepsilon)}\left(1+\frac{\gamma^{2}}{2 x}\right)\right]\left(F_{U U, T}+\varepsilon F_{U U, L}\right) \\
& \times\left\{1+\sqrt{2 \varepsilon(1+\varepsilon)} A_{U U}^{\cos \phi_{h}} \cos \phi_{h}+\varepsilon A_{U U}^{\cos \left(2 \phi_{h}\right)} \cos \left(2 \phi_{h}\right)+\lambda \sqrt{2 \varepsilon(1-\varepsilon)} A_{L U}^{\sin \phi_{h}} \sin \phi_{h}\right. \\
& +\frac{P_{\mathrm{T}}}{\sqrt{1-\sin ^{2} \theta \sin ^{2} \phi_{S}}}\left[\left(\underline{\cos \theta} A_{U T}^{\sin \left(\phi_{h}-\phi_{S}\right)}+\frac{1}{2} \sin \theta \sqrt{2 \varepsilon(1+\varepsilon)} A_{U L}^{\sin \phi_{h}}\right) \sin \left(\phi_{h}-\phi_{S}\right)\right. \\
& +\left(\underline{\cos \theta} \varepsilon A_{U T}^{\sin \left(\phi_{h}+\phi_{S}\right)}+\underline{\frac{1}{2}} \sin \theta \sqrt{2 \varepsilon(1+\varepsilon)} A_{U L}^{\sin \phi_{h}}\right) \sin \left(\phi_{h}+\phi_{S}\right) \\
& +\underline{\cos \theta \varepsilon A_{U T}^{\sin \left(3 \phi_{h}-\phi_{S}\right)} \sin \left(3 \phi_{h}-\phi_{S}\right)} \\
& +\underline{\cos \theta} \sqrt{2 \varepsilon(1+\varepsilon)} A_{U T}^{\sin \phi_{S}} \sin \phi_{S} \\
& +\left(\underline{\cos \theta} \sqrt{2 \varepsilon(1+\varepsilon)} A_{U T}^{\sin \left(2 \phi_{h}-\phi_{S}\right)}+\underline{\frac{1}{2}} \sin \theta \varepsilon A_{U L}^{\sin 2 \phi_{h}}\right) \sin \left(2 \phi_{h}-\phi_{S}\right) \\
& \left.+\underline{\frac{1}{2} \sin \theta \varepsilon A_{U L}^{\sin 2 \phi_{h}} \sin \left(2 \phi_{h}+\phi_{S}\right)}\right]
\end{aligned}
$$

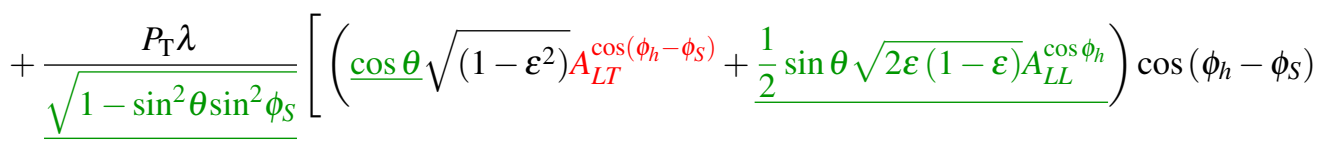

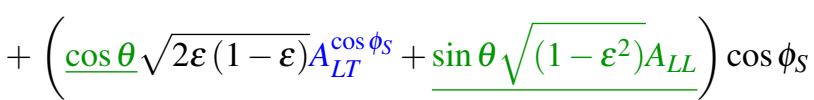

$$
\begin{aligned}
& +\left(\underline{\cos \theta} \sqrt{2 \varepsilon(1-\varepsilon)} A_{L T}^{\cos \left(2 \phi_{h}-\phi_{S}\right)}\right) \cos \left(2 \phi_{h}-\phi_{S}\right) \\
& \left.\left.+\frac{1}{2} \sin \theta \sqrt{2 \varepsilon(1-\varepsilon)} A_{L L}^{\cos \phi_{h}} \cos \left(\phi_{h}+\phi_{S}\right)\right]\right\}
\end{aligned}
$$

where $\varepsilon=\left(1-y-\frac{1}{4} \gamma^{2} y^{2}\right) /\left(1-y+\frac{1}{2} y^{2}+\frac{1}{4} \gamma^{2} y^{2}\right)$ and $\gamma=2 M x / Q$ and $\theta$ is the angle between beam and virtual photon momentum directions (see Figure 1). When compared with the "standard" crosssection [5], in which the effects due to the $P_{T}$ to $S_{T}$ transition have been neglected, 1.2 contains new ${ }^{1} \sin \theta$-scaled terms and $\theta$-depending factors and two extra azimuthal modulations $\left(\sin \left(2 \varphi_{h}+\varphi_{S}\right)\right.$ and $\cos \left(\varphi_{h}+\varphi_{S}\right)$ ) related to longitudinal amplitudes (these two amplitudes have been measured to be compatible with zero and are not going to be discussed in this letter).

Beside those two terms, the expression 1.2 counts in total eight more $w_{i}\left(\phi_{h}, \phi_{S}\right)$ azimuthal modulations. Each of this effects leads to a $A_{B T}^{w_{i}\left(\phi_{h}, \phi_{S}\right)}$ Transverse-Spin-dependent Asymmetry

\footnotetext{
${ }^{1}$ all "new" terms are underlined and marked in green.
} 
(TSA) defined as a ratio of the associated structure function $F_{B T}^{w_{i}\left(\phi_{h}, \phi_{S}\right)}$ to the azimuth-independent one $F_{U U}=F_{U U, T}+\varepsilon F_{U U, L}$. Here the superscript of the asymmetry indicates corresponding modulation, the first and the second subscripts - respective ("U"-unpolarized, "L"-longitudinal and "T"transverse) polarization of beam and target. Five amplitudes which depend only on target polarization are the target Single-Spin Asymmetries (SSA), the other three which depend also on $\lambda$ beam longitudinal polarization are known as Double-Spin Asymmetries (DSA).

As it can be seen from 1.2 in several cases TSAs are being mixed with $\sin \theta$-scaled longitudinal amplitudes. Since $\sin \theta$ is a rather small quantity in COMPASS kinematics [6] the influence of the additional to the TSAs terms, represented by $\sin \theta$-scaled longitudinal-spin amplitudes and $\theta$-angle dependent factors, is sizable only in the case of $A_{L T}^{\cos \varphi_{S}}$ DSA, which, even taking into account the smallness of $\sin \theta$, is still sizably affected by a large $A_{L L}$ amplitude [7]. To correct the $A_{L T}^{\cos \varphi_{S}}$ asymmetry we have used the $A_{L L}$ values evaluated based on [8]) which are in close agreement with the data $[6,7]$.

In the QCD parton model approach four of the eight transverse spin asymmetries $\left(A_{U T}^{\sin \left(\phi_{h}-\phi_{S}\right)}\right.$, $A_{U T}^{\sin \left(\phi_{h}+\phi_{S}\right)}, A_{U T}^{\sin \left(3 \phi_{h}-\phi_{s}\right)}$ SSAs and $A_{L T}^{\cos \left(\phi_{h}-\phi_{s}\right)}$ DSA) have Leading Order (LO) or leading-twist interpretation. The first two are the "Sivers" and "Collins" effects [9]-[11] which are the most studied ones. These asymmetries are given as convolutions of: $f_{1 T}^{\perp q}$ Sivers
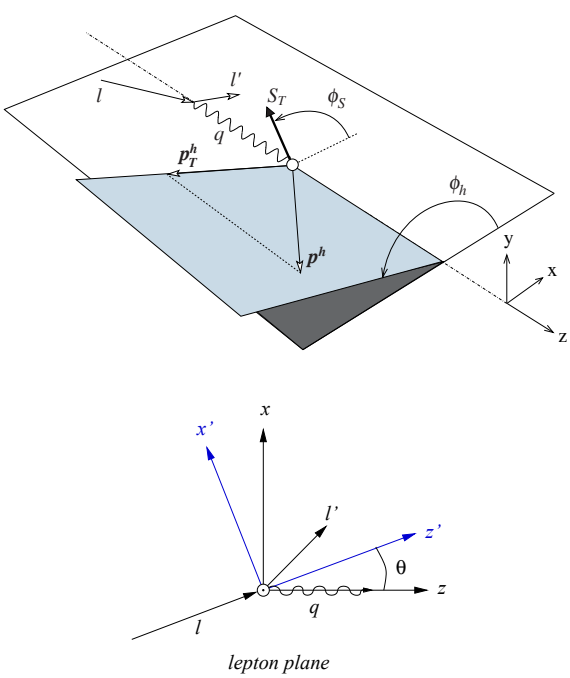

lepton plane

Figure 1: SIDIS process framework. PDF with $D_{1 q}^{h}$ ordinary FF, and $h_{1}^{q}$ "transversity" PDF with the $H_{1 q}^{\perp h}$ Collins FF, respectively. The other two LO terms are the $A_{U T}^{\sin \left(3 \phi_{h}-\phi_{S}\right)}$ single-spin asymmetry (related to $h_{1 T}^{\perp q}$ ("pretzelosity") PDF [12]-[17]) and $A_{L T}^{\cos \left(\phi_{h}-\phi_{S}\right)}$ DSA (related to $g_{1 T}^{q}$ ("worm-gear") distribution function [12]-[18],[8]).

Remaining four asymmetries are so-called "higher-twist" effects ${ }^{2}$. Corresponding structure functions enter at sub-leading order $\left(Q^{-1}\right)$ and contain terms given as various mixtures of twisttwo and twist-three (induced by quark-gluon correlations) parton distribution and fragmentation functions [2]-[20]. However, applying wildly used "Wandzura-Wilczek approximation" this higher twist objects can be simplified to the twist-two order (see [2,3] for more details). Complete list of "twist-two"-level interpretations for all eight TSAs is quoted in 1.2.

$$
\begin{aligned}
A_{U T}^{\sin \left(\phi_{h}-\phi_{S}\right)} & \propto f_{1 T}^{\perp q} \otimes D_{1 q}^{h}, A_{U T}^{\sin \left(\phi_{h}+\phi_{S}\right)} \propto h_{1}^{q} \otimes H_{1 q}^{\perp h}, \\
A_{U T}^{\sin \left(3 \phi_{h}-\phi_{S}\right)} \propto h_{1 T}^{\perp q} \otimes H_{1 q}^{\perp h}, A_{L T}^{\cos \left(\phi_{h}-\phi_{S}\right)} & \propto g_{1 T}^{q} \otimes D_{1 q}^{h} \\
A_{U T}^{\sin \left(\phi_{S}\right)} \propto Q^{-1}\left(h_{1}^{q} \otimes H_{1 q}^{\perp h}+f_{1 T}^{\perp q} \otimes D_{1 q}^{h}\right), A_{U T}^{\sin \left(2 \phi_{h}-\phi_{S}\right)} & \propto Q^{-1}\left(h_{1 T}^{\perp q} \otimes H_{1 q}^{\perp h}+f_{1 T}^{\perp q} \otimes D_{1 q}^{h}\right), \\
A_{L T}^{\cos \left(\phi_{S}\right)} \propto Q^{-1}\left(g_{1 T}^{q} \otimes D_{1 q}^{h}\right), A_{L T}^{\cos \left(2 \phi_{h}-\phi_{S}\right)} & \propto Q^{-1}\left(g_{1 T}^{q} \otimes D_{1 q}^{h}\right) .
\end{aligned}
$$

The whole set of eight SIDIS asymmetries has been already measured at COMPASS for both deuteron and proton targets (See [9]-[17] and references therein).

\footnotetext{
${ }^{2}$ in equations 1.2-1.4 the twist- 2 amplitudes are marked in red and higher-twist ones in blue
} 
Using similar notations, single-polarized $\left(\pi N^{\uparrow}\right)$ Drell-Yan cross-section at leading order can be written in the following model-independent way [21]:

$$
\begin{aligned}
\frac{d \sigma^{L O}}{d \Omega}=\frac{\alpha_{e m}^{2}}{F q^{2}} F_{U}^{1}\{1 & +\cos ^{2} \theta+\sin ^{2} \theta A_{U}^{\cos 2 \varphi_{C S}} \cos 2 \varphi_{C S}+S_{T}\left[\left(1+\cos ^{2} \theta\right) A_{T}^{\sin \varphi_{S}} \sin \varphi_{S}\right. \\
& \left.\left.+\sin ^{2} \theta\left(A_{T}^{\sin \left(2 \varphi_{C S}+\varphi_{S}\right)} \sin \left(2 \varphi_{C S}+\varphi_{S}\right)+A_{T}^{\sin \left(2 \varphi_{C S}-\varphi_{S}\right)} \sin \left(2 \varphi_{C S}-\varphi_{S}\right)\right]\right)\right\} .
\end{aligned}
$$

Here $\varphi_{C S}$ and $\varphi_{S}$ angular variables are defined in "Collins-Soper" and "target rest" frames, correspondingly (see Figure 2). Similarly to the SIDIS case, "U", "L" and "T" subscripts denote the state of the target polarization while the superscript indicates the corresponding modulation.

As one can see, at LO the DY cross-section contains only one unpolarized and three target transverse spin dependent azimuthal asymmetries. Within the same QCD parton model approach, at variance with the SIDIS-case, Drell-Yan asymmetries are interpreted as convolutions of two TMD PDFs, one of the the "beam" and one of the "target" hadron. Quoting only the target nucleon PDFs: the $A_{T}^{\sin \varphi_{s}}, A_{T}^{\sin \left(2 \varphi_{C S}-\varphi_{s}\right)}$ and $A_{T}^{\sin \left(2 \varphi_{C S}+\varphi_{s}\right)}$ give access to the "Sivers" $f_{1 T}^{\perp q}$, "transversity" $h_{1}^{q}$ and "pretzelosity" $h_{1 T}^{\perp q}$, distribution functions, respectively. Within the QCD-concept of generalized universality of TMD PDFs it appears that same parton distribution functions can be accessed both in SIDIS and Drell-Yan (see the Table. 1 for the complete list). Therefore, future COMPASS results on DY asymmetries are intriguingly complementary to the results previously obtained by the same collaboration for azimuthal effects in SIDIS. The comparison of two sets
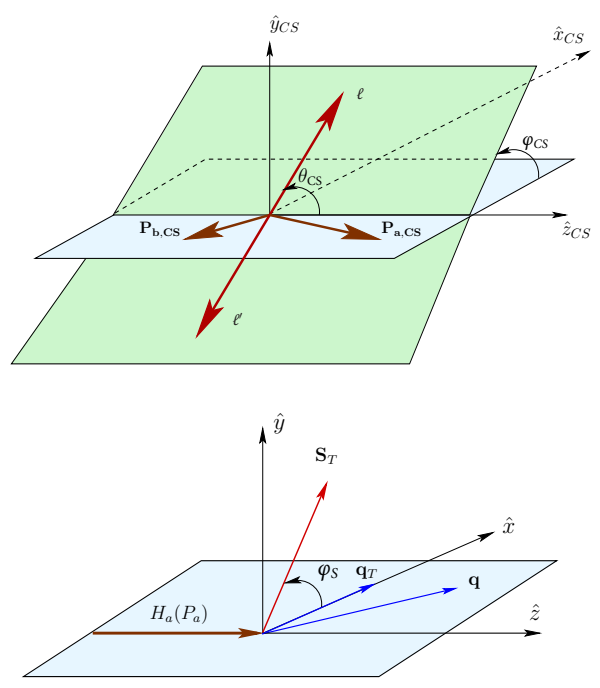
will give an unprecedented opportunity to access TMD

PDFs via two mechanisms and test their universality and key features (for instance, predicted Sivers and Boer-Mulders PDFs sign change) using essentially same experimental setup.

\begin{tabular}{ccc}
\hline SIDIS $\ell^{\rightarrow} N^{\uparrow}$ & TMD PDF & DY $\pi N^{\uparrow}($ LO) \\
\hline$A_{U U}^{\cos 2 \phi_{h}}, A_{U U}^{\cos \phi_{h}}$ & $h_{1}^{\perp q}$ & $A_{U}^{\cos 2 \varphi_{C S}}$ \\
\hline$A_{U T}^{\sin \left(\phi_{h}-\phi_{s}\right)}, A_{U T}^{\sin \phi_{s}}, A_{U T}^{\sin \left(2 \phi_{h}-\phi_{s}\right)}$ & $f_{1 T}^{\perp q}$ & $A_{T}^{\sin \varphi_{S}}$ \\
\hline$A_{U T}^{\sin \left(\phi_{h}+\phi_{s}-\pi\right)}, A_{U T}^{\sin \phi_{s}}$ & $h_{1}^{q}$ & $A_{T}^{\sin \left(2 \varphi_{C S}-\varphi_{S}\right)}$ \\
\hline$A_{U T}^{\sin \left(3 \phi_{h}-\phi_{s}\right)}, A_{U T}^{\sin \left(2 \phi_{h}-\phi_{s}\right)}$ & $h_{1 T}^{\perp q}$ & $A_{T}^{\sin \left(2 \varphi_{C S}+\varphi_{S}\right)}$ \\
\hline$A_{L T}^{\cos \left(\phi_{h}-\phi_{s}\right)}, A_{L T}^{\cos \phi_{s}}, A_{L T}^{\cos \left(2 \phi_{h}-\phi_{s}\right)}$ & $g_{1 T}^{q}$ & double-polarized DY \\
\hline
\end{tabular}

Table 1: Nucleon TMD PDFs accessed via SIDIS and Drell-Yan azymmetries.

Another hot topic being addressed by the COMPASS collaboration is the multi-differential analysis of SIDIS data. In general, asymmetries being represented as convolutions of different 
TMD distribution functions are considered to be complex objects a priori dependent on the experimental choice of multidimensional kinematical phase-space. Thus, in order to reveal the most complete multivariate dependence of TMD PDFs, it is important to extract azimuthal amplitudes as multi-differential functions of kinematical variables. In practice, available experimental data often are too limited for such an ambitious approach and studying dependence of the asymmetries on some specific kinematic variable one is forced to stick to one-dimensional case integrating over all the other variables.

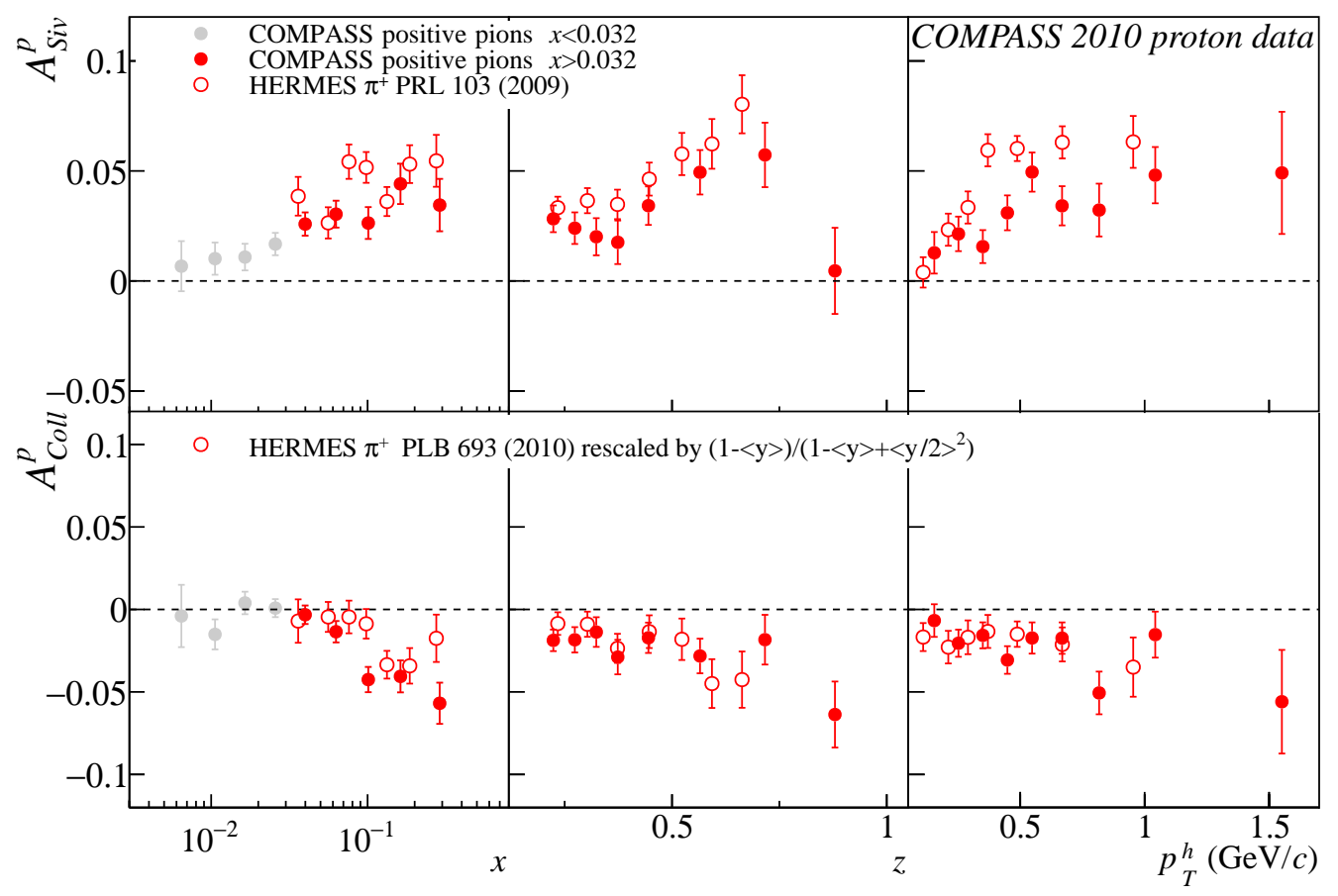

Figure 3: Sivers (top) and Collins (bottom) asymmetries at COMPASS and HERMES.

Presently, one of the related challenges in the field of spin-physics is the study of TMD evolution of various PDFs and FFs and related asymmetries. Comparison of COMPASS and HERMES results for Sivers and Collins asymmetries on proton [9, 22, 23] emphasized the importance of this domain. In the Figure 3 are demonstrated Sivers (top) and Collins (bottom) asymmetries for positive hadrons as measured at COMPASS and HERMES experiments. While results for the Collins asymmetries appear to be compatible, Sivers effect at COMPASS at large $x$ is noticeably smaller than the one obtained by HERMES. Here the important detail is that at given $x$ COMPASS $Q^{2}$ values are by a factor of $3-4$ larger than the HERMES ones. Thus, observed behaviour of Sivers and Collins effects can be used to adjust description of the $Q^{2}$-dependence of TMDs. Presently different models predict from small up to quite large QCD-evolution effects attempting to describe available experimental observations and make predictions for the future ones [24]-[26]. Additional precise experimental measurements exploring different $Q^{2}$ domains for fixed $x$-range are necessary to further constrain the theoretical models. The work described in this review is a unique and first ever attempt to explore behaviour of TSAs in the multivariate kinematical environment of the data collected by a single experiment. For this purpose COMPASS experimental data was split into five 
different $Q^{2}$ ranges giving an opportunity to study asymmetries as a function of $Q^{2}$ at fixed bins of $x$. Additional variation of $z$ and $p_{T}$ cuts allows to deeper explore multi-dimensional behaviour of the TSAs and their TMD constituents.

\section{Multidimensional analysis of TSAs}

During the "phase-1" (from 2002 to 2010) COMPASS has performed series of SIDIS datatakings using $160 \mathrm{GeV} / \mathrm{c}$ longitudinally polarized muon beam and transversely polarized ${ }^{6} \mathrm{LiD}$ and $\mathrm{NH}_{3}$ targets (See [10]-[17] and references therein). In 2012 COMPASS entered in "phase-II" and recently performed Drell-Yan measurements with $190 \mathrm{GeV} / \mathrm{c} \pi^{-}$beam and unpolarized (in 2014) and transversely polarized $\mathrm{NH}_{3}$-targets (in 2015) [14, 21].

Very soon both sets of COMPASS results from SIDIS and Drell-Yan will become a subject of global fits and phenomenological analyses. In order to do provide relevant input for these studies, COMPASS SIDIS proton 2010 data has been re-analyzed in a more differential way extracting the asymmetries in the same four $Q^{2}$ kinematic regions which were selected for the COMPASS Drell-Yan measurement program [14, 21]: $Q^{2} /(\mathrm{GeV} / \mathrm{c})^{2} \in[1 ; 4],[4 ; 6.25],[6.25 ; 16],[16 ; 81]$. Preliminary results obtained with this selection have been presented in $[12,14]$ while current review is dedicated to more recent $x-z-p_{T}-Q^{2}$ multi-dimensional approach [13].

The analysis was carried out on the same data-sample collected in 2010 with transversely polarized proton target. General event selection as well as asymmetry extraction and systematic uncertainty evaluation procedures were identical to those used for recent COMPASS results on Collins, Sivers and other TSAs [10]-[17].
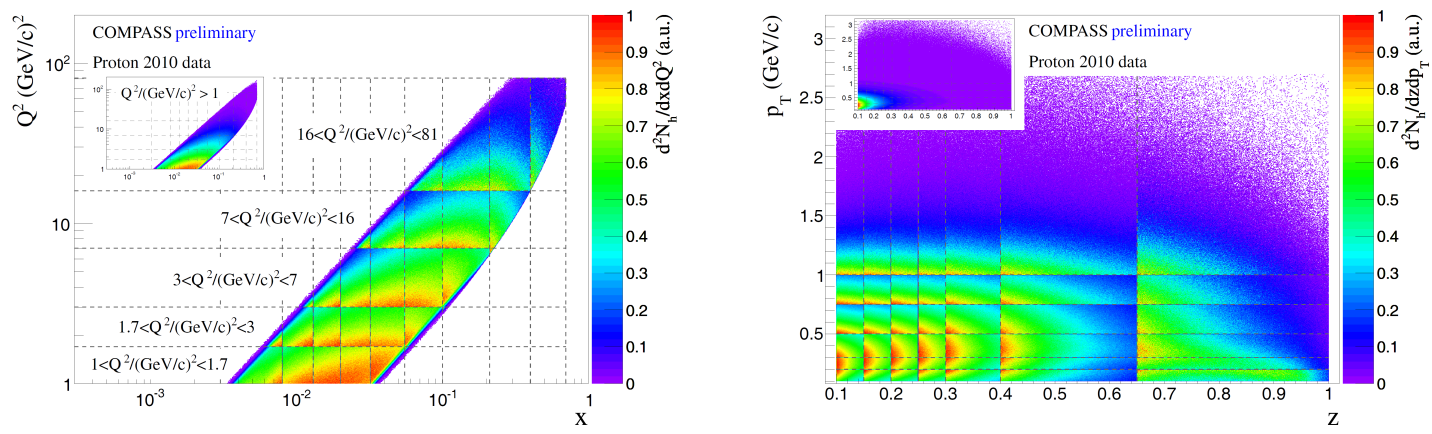

Figure 4: COMPASS $x: Q^{2}$ (left) and $z: p_{T}$ (right) phase space coverage.

Amplitudes for all ten azimuthal modulations present in the transverse spin dependent part of the cross-section 1.2 have been extracted simultaneously using extended unbinned maximum likelihood estimator. Obtained "raw" asymmetries have been then corrected for average depolarization factors ( $\varepsilon$-depending factors in equation 1.2 standing in front of the amplitudes), dilution factor and target and beam (only DSAs) polarizations evaluated in the given kinematical bin [10]-[17]. Primary sample is defined by the following standard DIS cuts: $Q^{2}>1(\mathrm{GeV} / \mathrm{c})^{2}, 0.003<x<0.7$ and $0.1<y<0.9$ and two more hadronic selections: $p_{T}>0.1 \mathrm{GeV} / \mathrm{c}$ and $z>0.1$.

In order to study possible $Q^{2}$-dependence the $x: Q^{2}$ phase-space covered by COMPASS experimental data has been divided into $5 \times 9$ two-dimensional grid (see left plot in Figure 4). where 
five $Q^{2}$-ranges are the following ones: $Q^{2} /(\mathrm{GeV} / \mathrm{c})^{2} \in[1 ; 1.7],[1.7 ; 3],[3 ; 7],[7 ; 16],[16 ; 81]$. In addition, each of this samples has been divided into five $z$ and five $p_{T}(\mathrm{GeV} / \mathrm{c})$ sub-ranges defined as follows:

$z>0.1, z>0.2,0.1<z<0.2,0.2<z<0.4$ and $0.4<z<1.0$

$p_{T}>0.1,0.1<p_{T}<0.75,0.1<p_{T}<0.3,0.3<p_{T}<0.75$ and $p_{T}>0.75$.

Using various combinations of aforementioned cuts and sub-ranges, asymmetries have been studied in following "3D" and "4D" configurations: 1) $x$-dependence in $Q^{2}-z$ and $Q^{2}-p_{T}$ grids. 2) $Q^{2}$-dependence in $x-z$ and $x$ - $p_{T}$ grids. 3) $Q^{2}$ - (or $x$-) dependence in $x$ - $p_{T}$ (or $Q^{2}-p_{T}$ ) grids with different choices of $z$ sub-range.

The second general approach was defined to focus on $z$ - and $p_{T}$-dependences in different $x$ ranges. For this study, two-dimensional $z: p_{T}$ phase-space has been divided into $7 \times 6$ grid as it is demonstrated in the right plot in Figure 4. Selecting in addition three different $x$-ranges: $0.003<$ $x<0.7,0.003<x<0.032,0.032<x<0.7$, asymmetries have been extracted in "3D: $x-z-p_{T}$ " grids. In the next section selected COMPASS preliminary results obtained for multi-dimensional target transverse spin dependent azimuthal asymmetries are presented.

\section{Results}

${ }^{3}$ As an example of "3D" Sivers and Collins effects, results for the extracted $x-z-Q^{2}$ configurations are presented in the Figure 5 ('top' and 'bottom' plots, respectively). As a general observation, for positive hadron production Sivers asymmetry shows sizable signal along whole $x$-range, while for negative hadrons effect is not clear. Still, there are some indications for a positive Sivers signal at relatively large $x$ and $Q^{2}$ and for a negative effect at low $x$. Clear "mirrored" behaviour for positive and negative hadron amplitudes is being observed in most of the bins for Collins effect. In general, both Sivers and Collins amplitudes tend to increase in absolute value with $z$ and $p_{T}$.

Demonstrated in the Figure $5 Q^{2}$-dependences of Sivers and Collins asymmetries serve as a direct input for TMD-evolution related studies. In fact, for Sivers effect in several $\mathrm{x}$-bins there are some hints for possible decreasing $Q^{2}$-dependence for positive hadrons which become more evident at large $z$. In the meantime, Collins asymmetry does not show any clear indications for $Q^{2}$ dependence. Thus, both these observations are in agreement with quoted previously COMPASSHERMES comparison for Sivers and Collins effects (see [9, 22, 23] and Figure 3).

Another SSA which is found to be non-zero at COMPASS is the $A_{U T}^{\sin \left(\phi_{s}\right)}$ term which is presented in Figure 6 (top) in "3D: $x-z-p_{T}$ " configuration. Here the most interesting is the large $z$-range were amplitude is measured to be sizable and non zero both for positive and negative hadrons. It is relevant to remind that within the "Wandzura-Wilczek approximation" this asymmetry can be associated with Sivers and Collins mechanisms.

The bottom plot in the Figure 6 is dedicated to the $A_{L T}^{\cos \left(\phi_{h}-\phi_{S}\right)}$ DSA explored in "3D: $Q^{2}$ $z-x "$ grid and superimposed with the theoretical curves from [18]. This is the only DSA which appears to be non-zero at COMPASS and the last TSA for which a statistically significant signal has been detected. Remaining four asymmetries are found to be small or compatible with zero within available statistical accuracy which is in agreement with available predictions [19, 20, 28]. As an example the "3D: $x-z-p_{T}$ " results for $A_{U T}^{\sin \left(3 \phi_{h}-\phi_{S}\right)}$ asymmetry are presented in Figure 7.

\footnotetext{
${ }^{3}$ Results discussed in this section have been first presented at the SPIN-2014 conference [13], see also [5],[27].
} 


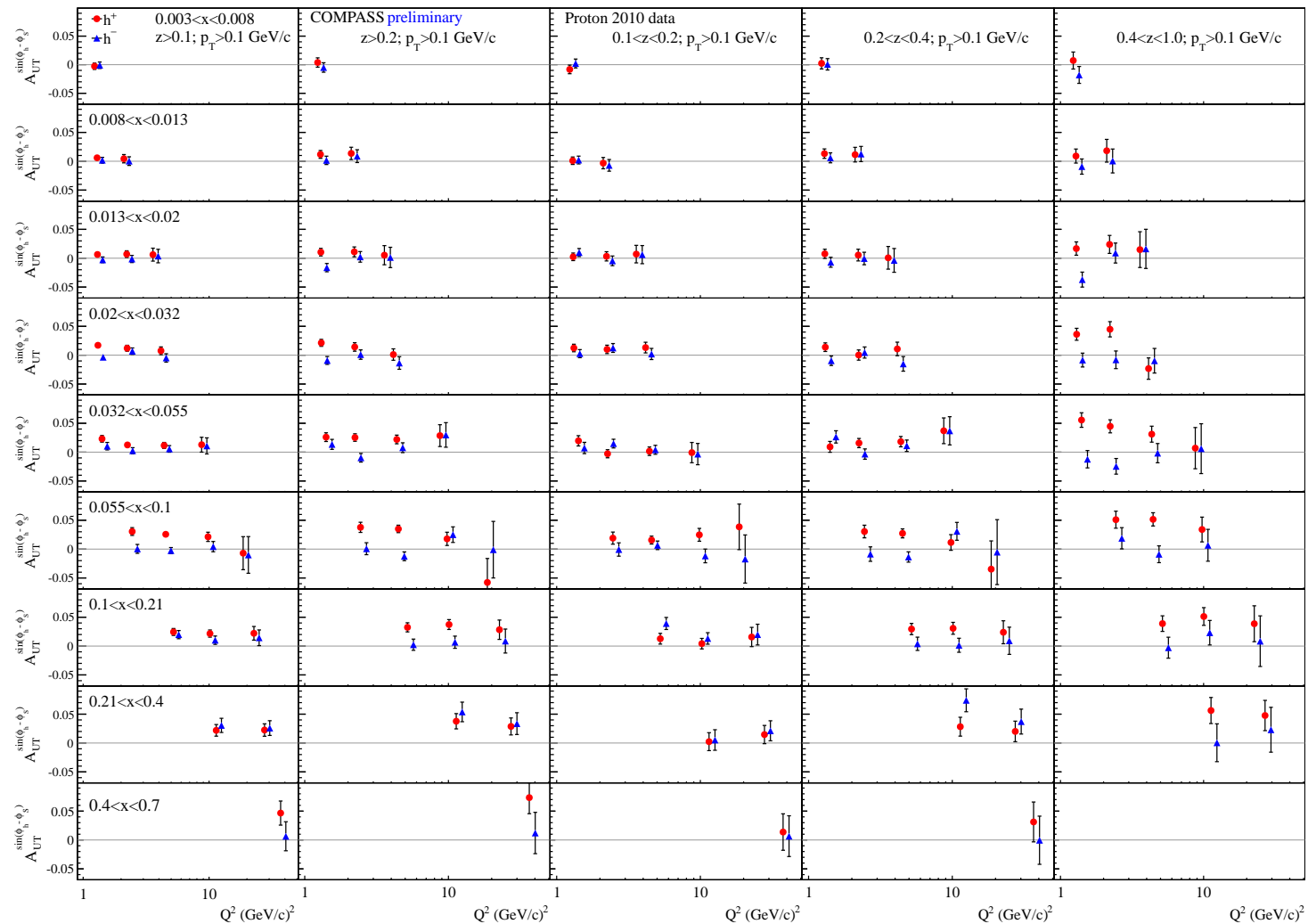

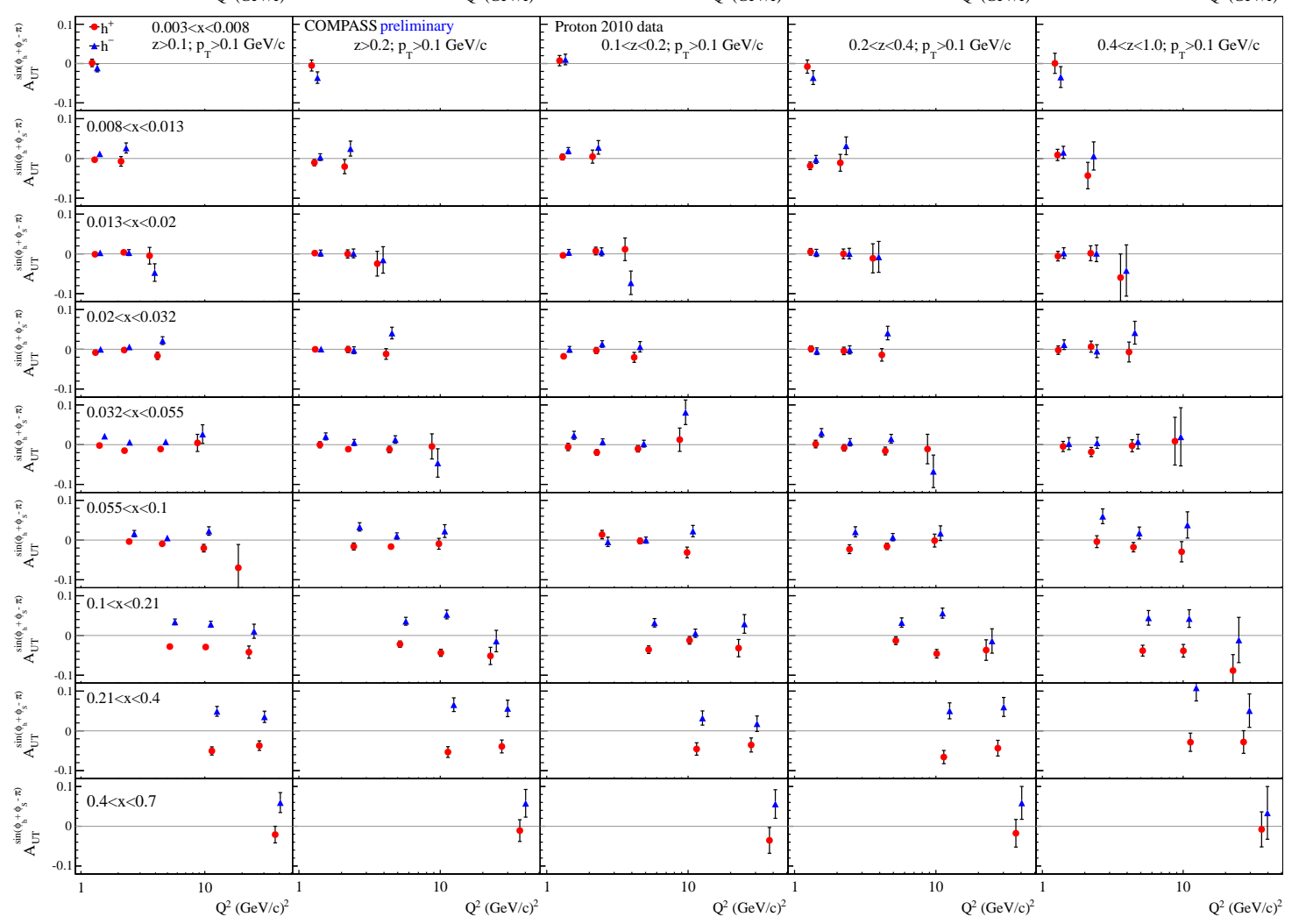

Figure 5: Sivers (top) and Collins (bottom) asymmetries in "3D" $x-z-Q^{2}$. 

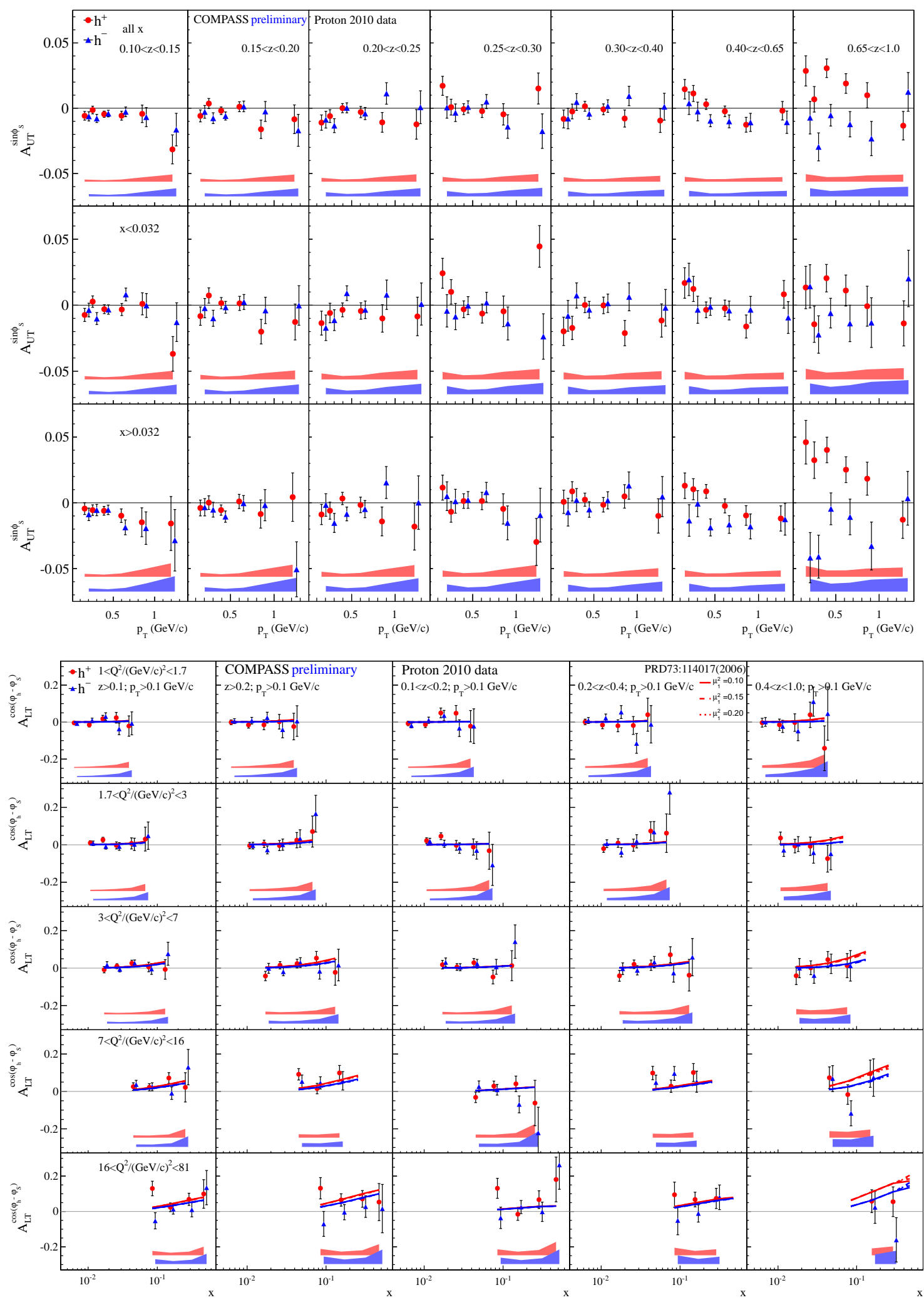

Figure 6: Top: $A_{U T}^{\sin \left(\phi_{s}\right)}$ asymmetry in "3D" $\left(x-z-p_{T}\right)$. Bottom: $A_{L T}^{\cos \left(\phi_{h}-\phi_{S}\right)}$ in "3D" $\left(Q^{2}-z-x\right)$ superimposed with theoretical predictions from [18]. 


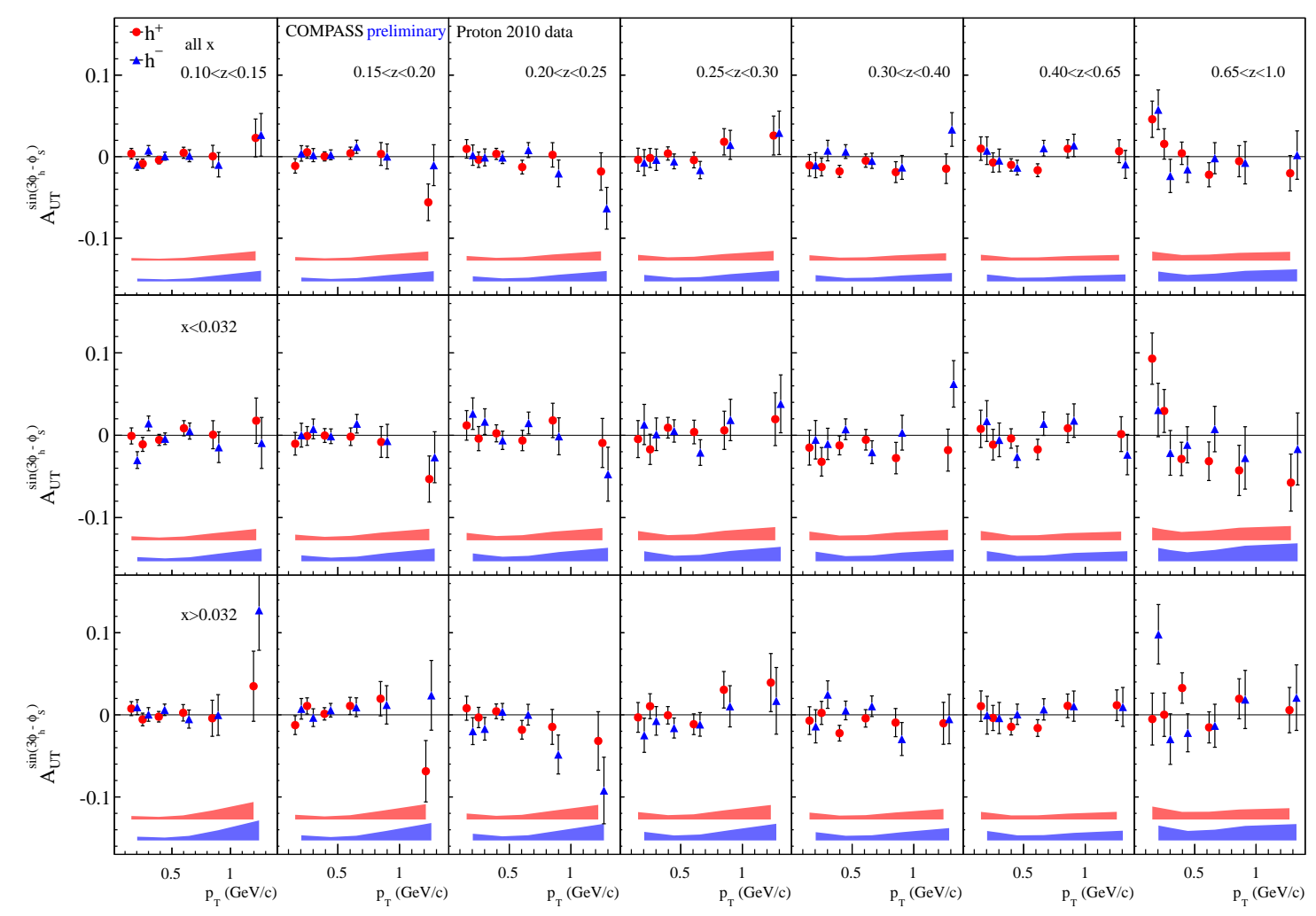

Figure 7: $A_{U T}^{\sin \left(3 \phi_{S}-\phi_{S}\right)}$ asymmetry in "3D" $\left(x-z-p_{T}\right)$.

\section{Conclusions}

COMPASS multidimensional analysis of the whole set of proton TSAs has been performed exploring various multi-differential configurations in the $x: Q^{2}: z: p_{T}$ phase-space. Particular attention was focused on the possible $Q^{2}$-dependence of asymmetries, serving a direct input to TMDevolution related studies. Several interesting observations have been made studying the results obtained for Sivers, Collins, $A_{L T}^{\cos \left(\phi_{h}-\phi_{S}\right)}$ and $A_{U T}^{\sin \left(\phi_{S}\right)}$ asymmetries. Other four asymmetries were found to be compatible with zero within available statistical accuracy.

This is the first ever attempt to extract multi-differential dependencies of all possible transverse spin dependent asymmetries using experimental data collected by a single experiment. Provided highly differential data set, combined with past and future relevant data of other collaborations, will give a unique opportunity to access the whole set of TMD PDFs and test their multi-differential nature.

Also particularly interesting will be the planned comparison of presented SIDIS TSAs with the Drell-Yan asymmetries which soon will be extracted from first ever polarized Drell-Yan data collected by COMPASS in 2015. This unique opportunity to explore nucleon spin-structure via two different processes measured with the same experimental setup, will be the first direct chance to test the universality and key features of TMD PDFs such as, for instance, expected "sign change" of the Sivers function. 


\section{References}

[1] A. Kotzinian, Nucl. Phys. B 441, 234 (1995) [ arXiv: hep-ph/9412283].

[2] P. J. Mulders and R. D. Tangerman, Nucl. Phys. B 484, 538 (1997) [ arXiv: hep-ph/9510301].

[3] A. Bacchetta et al. JHEP 0702, 093 (2007) [ arXiv: hep-ph/ 0 611265].

[4] M. Diehl and S. Sapeta, Eur. Phys. J. C 41, 515 (2005) [ arXiv: hep-ph/ 0503023 ].

[5] B. Parsamyan, CIPANP2015 Conference Proceedings [ arXiv:1511.09093]

[6] B. Parsamyan, Phys. Part. Nucl. 45, 158 (2014) [ arXiv:1301. 6615].

[7] M. G. Alekseev et al. Phys. Lett. B 693 (2010) 227, [arXiv: 1007 . 4061 ].

[8] M. Anselmino et al. Phys. Rev. D 74, 074015 (2006) [ arXiv: hep-ph/ 0608048 ].

[9] C. Adolph et al. Phys. Lett. B $\mathbf{7 4 4} 250$ (2015) [ arXiv:1408.4405].

[10] C. Adolph et al. Phys. Lett. B 717, 376 (2012) [ arXiv:1205.5121].

[11] C. Adolph et al. Phys. Lett. B 717, 383 (2012) [ arXiv:1205. 5122].

[12] B. Parsamyan, EPJ Web Conf. 85 (2015) 02019 [ arXiv: 1411. 1568].

[13] B. Parsamyan, TBP in Int. J. Mod. Phys. Conf. Ser. [ arXiv:1504.01599].

[14] B. Parsamyan, TBP in Int. J. Mod. Phys. Conf. Ser. [ arXiv:1504.01598].

[15] B. Parsamyan, PoS DIS 2013, 231 (2013) [ arXiv: 1307.0183 ].

[16] B. Parsamyan, J. Phys. Conf. Ser. 295, 012046 (2011) [ arXiv: 1012 . 0155].

[17] B. Parsamyan, Eur. Phys. J. ST 162, 89 (2008) [ arXiv: 0709.3440 ].

[18] A. Kotzinian et al. Phys. Rev. D 73, 114017 (2006) [ arXiv: hep-ph/0603194].

[19] W. Mao, Z. Lu and B. Q. Ma, Phys. Rev. D 90 (2014) 1, 014048 [ arXiv: 1405.3876 ].

[20] W. Mao et al. Phys. Rev. D 91 (2015) 3, 034029 [ arXiv:1412 . 7390].

[21] F. Gautheron et al. "COMPASS-II Proposal” CERN-SPSC-2010-014.

[22] A. Airapetian et al. Phys. Rev. Lett. 103 (2009) 152002 [ arXiv: 0906.3918 ].

[23] A. Airapetian et al. Phys. Lett. B 693 (2010) 11 [ arXiv: 1006.4221 ].

[24] S. Aybat, A. Prokudin and T. Rogers, Phys. Rev. Lett. 108 (2012) 242003 [ arXiv:1112 . 4423].

[25] M. G. Echevarria et al. Phys. Rev. D 89, 074013 (2014) [ arXiv: 1401 . 5078].

[26] P. Sun and F. Yuan, Phys. Rev. D 88, no. 11, 114012 (2013) [ arXiv: 1308 . 5003].

[27] B. Parsamyan, DSPIN2015 Conference Proceedings [ arXiv:1512.06772].

[28] C. Lefky and A. Prokudin, Phys. Rev. D 91 (2015) 3, 034010 [ arXiv: 1411.0580 ]. 